\title{
Zur Veräußerung der ägyptischen Sammlung von Prof. Dr. Georg Steindorff an die Universität Leipzig 1936/37
}

\author{
Verwaltungsgericht Berlin, Urteil vom 26. Mai 2011 - 29 K 126/09
}

1. Eine bewusste Veräußerung unter Wert durch einen Verfolgten des Nationalsozialismus stellt nicht ohne Weiteres eine gemischte Schenkung dar, da sonst die Verfolgungsvermutung nach Art. 3 Abs. 2 REAO ausgehebelt würde, soweit sie den Beweis eines angemessenen Kaufpreises erfordert. Der Beweis der (Teil-) Schenkungsabsicht obliegt dem Verfügungsberechtigten.

2. Es reicht nicht aus, ein schlüssiges Bild einer verfolgungsneutralen Veräußerung darzustellen, da dies nur einen Anscheinsbeweis erschüttern könnte, nicht aber die Verfolgungsvermutung des Art. 3 Abs. 3 REAO widerlegen kann.

\section{Tatbestand}

- Die klagende Universität wendet sich gegen die Rückübertragung der noch vorhandenen näher bezeichneten 163 Stücke der altägyptischen Sammlung aus dem früheren Eigentum von Prof. Dr. Georg Steindorff an die Beigeladene.

Steindorff war jüdischer Abstammung, seit 1893 außerordentlicher Professor an der Universität Leipzig und von 1904 bis zu seiner Emeritierung zum 31. März 1934 Inhaber des dortigen Lehrstuhls für Ägyptologie. In dieser Zeit legte er sich, teils bei eigenen Forschungsreisen, besagte Sammlung zu. 1936 bot er die zum Teil bereits als Leihgabe im Ägyptischen Museum der Universität befindliche Sammlung der Universität für 8.000 RM zum Kauf an, andernfalls er einen Verkauf an das Kestner-Museum in Hannover erwäge. Sein Nachfolger Prof. Dr. W. schrieb am 21. August 1936 an das Sächsische Ministerium für Volksbildung, Steindorff habe früher die Absicht gehabt, seine Sammlung dereinst der Universität zu schenken, daran aber wegen der Änderung der wirtschaftlichen Verhältnisse nicht mehr festhalten können; den Kaufpreis könne er nur als mäßig bezeichnen. Einem Schreiben von W. vom 28. Oktober 1936 an den Rektor der Universität Leipzig zu Folge gab Steindorff an, die gleiche Summe zu verlangen, die er selbst aufgewendet habe; er habe die Sammlung immer als eine Art Sparbüchse betrachtet und nie mit einer Verzinsung gerechnet. Steindorff habe den Wert auf 10.260 RM geschätzt, er selbst - W. - schätze ihn noch weit höher. Der Ankauf wurde am 1. März 1937 genehmigt, der Kaufpreis wurde in drei Raten zu 2.000 RM und 2 × 3.000 RM gezahlt.

Die Beklagte hat einige Literaturstellen ermittelt, wonach Steindorff die Sammlung „für einen Pappenstiel“ an die Universität verkauft habe. Auch die spätere Leiterin des Museums B. bezeichnete den Preis 1979 als "schäbig”.

Mit Schreiben vom 9. Dezember 1938 teilte Steindorff dem Universitäts-Rentamt mit, dass er beabsichtige, seinen Wohnsitz ins Ausland zu verlegen, und bat, die letzte Rate an den für die Auswanderung beauftragten Spediteur zu überweisen. Mangels förmlicher Abtretung wurde der Betrag Anfang
1940 zunächst auf ein Sperrkonto überwiesen, von wo sie der Schwiegersohn von Steindorff zur Zahlung an den bei der Auswanderung beauftragten Spediteur erhalten sollte.

Das Verhältnis von Steindorff zu W. scheint - auch nach Steindorffs eigenen Aussagen - bis zur Ausreise ein gutes gewesen zu sein; so überließ W. ihm weiterhin ein Arbeitszimmer in der Universität. Anlässlich der Ausreise kam es dann zu einem Zerwürfnis über die Frage, ob bestimmte Unterlagen Privateigentum von Steindorff oder Universitätseigentum waren. Unmittelbar nach Kriegsende machte Steindorff Nach- und Fortzahlung seiner Pension sowie Rückgabe aus seiner Sicht privater Unterlagen geltend, nicht aber Rückgabe seiner Sammlung.

Steindorff verstarb 1951 in den USA. Zuvor hatte er noch einen Briefwechsel mit dem Leiter der Sammlung, Dr. M., in dem er sich erfreut über die Wiedereröffnung der Sammlung und die Würdigung seiner Tätigkeit äußerte.

Im Dezember 1958 meldete die Witwe von Steindorff rückerstattungsrechtliche Ansprüche wegen Silbersachen, Schmuckgegenständen, Hausrat und wissenschaftlicher Fachliteratur an. Für die Literatur erhielt sie im Vergleichswege 3.000,- DM, im Übrigen wurde der Antrag abgelehnt, da nicht nachzuweisen war, dass die Gegenstände in den Geltungsbereich des Bundesrückerstattungsgesetzes gelangt waren.

Mit Schreiben vom 9. Dezember 1995 konkretisierte die Beigeladene ihre Anmeldung vom 22. Dezember 1992 (ANM-3) auf die "Ägyptische Sammlung (heute im Bestand der Universität Leipzig) von Prof. Georg Steindorff". Sie ist im Bestand der Devisenstelle im Staatsarchiv Leipzig benannt. Im Jahre 2007 meldete sich H., ein Enkel von Steindorff, und erklärte, die Sammlung sei de facto bereits 1908 der Universität überlassen („donated“) worden, und es sei unangemessen, sie jetzt der Universität zu entziehen und an den legitimen Erben vorbei Fremden zukommen zu lassen. Er bat, ihm den Anspruch zuzuerkennen, damit er darauf zu Gunsten der Universität verzichten könne.

Mit Teilbescheid vom 3. Juli 2009 übertrug das Bundesamt für zentrale Dienste und offene Vermögensfragen die Sammlung auf die Beigeladene zurück und lehnte den Antrag von $\mathrm{H}$. 
ab. Die Anmeldung der Beigeladenen sei wirksam, da das Eigentum des Verfolgten in den Akten des Rückerstattungsverfahrens der Oberfinanzdirektion Berlin und in den Akten des Staatsarchivs Leipzig nachgewiesen sei. Die Anmeldung von $\mathrm{H}$. sei verspätet. Die Verfolgungsvermutung sei nicht widerlegt, da der Geschädigte zwar über den Kaufpreis frei habe verfügen können, aber selbst den Wert seiner Sammlung höher als den Kaufpreis eingeschätzt habe. Für den Entschluss, die Sammlung nicht, wie zunächst geplant, der Universität zu schenken, sondern sie zu verkaufen, sei der Verfolgungs- und Auswanderungsdruck jedenfalls mitursächlich gewesen.

Mit der am 24. Juli 2009 bei Gericht eingegangenen Klage macht die Klägerin geltend, Steindorff sei nicht verfolgt worden, wie sich daran zeige, dass er bis zu seiner Ausreise ein Arbeitszimmer in der Universität habe nutzen dürfen sowie publizieren können. Zu seinem 75. Geburtstag sei er von hochgestellten Persönlichkeiten und in zahlreichen Veröffentlichungen, darunter ansonsten judenfeindlichen Tageszeitungen, gewürdigt worden. Ebenfalls hochgestellte Personen hätten sich schließlich dafür eingesetzt, ihm seine Ausreise zu ermöglichen. Der Verkauf sei demnach nicht verfolgungsbedingt erfolgt, zumal Steindorff weiter seine Pension bezogen habe und nicht auf Geld angewiesen gewesen sei. Der Verkauf habe vielmehr dazu gedient, seinem nichtjüdischen Schwiegersohn, der sein Rauchwarenkommissionsgeschäft in Folge der Wirtschaftskrise habe einstellen müssen, einen geschäftlichen Neuanfang zu ermöglichen. Dabei habe Steindorff vor Allem ein Interesse daran gehabt, seine Sammlung der Universität Leipzig zukommen zu lassen. Lediglich wegen der Wirtschaftskrise habe er überhaupt Geld verlangt. Auf den Betrag sei es ihm nicht angekommen, da er die Sammlung ansonsten auch mindestens zu dem von ihm geschätzten Wert hätte verkaufen können. Außer der Universität Leipzig seien auch das Kestner-Museum oder die Universitäten München oder Göttingen als Käufer in Betracht gekommen. Der Preis sei nicht zu niedrig gewesen, da bei dem Verkauf ganzer Sammlungen entsprechende Abschläge gegenüber der Summe der Einzelwerte damals wie heute üblich seien. Frau B. halte an ihrer Einschätzung von 1979 nicht fest. Da es Steindorff darauf angekommen sei, seine Sammlung der Universität zukommen zu lassen, wäre zudem das Geschäft auch ohne die Herrschaft des Nationalsozialismus erfolgt. Schließlich verdeutlichten die Bemühungen staatlicher Stellen, dem jüdischen Emigranten die dritte Rate des Kaufpreises zukommen zu lassen, dass das Rechtsgeschäft unabhängig vom Nationalsozialismus zu Stande gekommen sei.

Dies werde zudem dadurch belegt, dass er und seine Frau nach dem Krieg Restitutionsansprüche nur für sonstige Vermögenswerte, nicht aber für die Sammlung angemeldet hätten. Weiterhin zeigten seine im Briefwechsel mit M. geäußerten Glückwünsche zur Wiederaufstellung der Sammlung, dass deren Verbleib seinen Wünschen entsprochen habe. Jedenfalls sei darin ein Verzicht auf Restitutionsansprüche zu sehen.
Die Klägerin beantragt,

Nr. 1 des Teilbescheides des Bundesamtes für zentrale Dienste und offene Vermögensfragen vom 3. Juli 2009 aufzuheben.

Die Beklagte beantragt,

die Klage abzuweisen.

Die Verfolgungsvermutung sei nicht widerlegt. Dass es Steindorff freigestanden habe, die Sammlung zu einem höheren Preis zu veräußern, sei nicht belegt, insbesondere kein höheres Gebot des Kestner-Museums. Auch sei der Kaufpreis nicht frei verfügbar gewesen, da die letzte Rate auf ein Sperrkonto gezahlt worden sei. Schließlich sei es zwar wahrscheinlich, dass er die Sammlung auch ohne die Herrschaft des Nationalsozialismus der Universität überlassen hätte, doch schließe dies die Mitursächlichkeit des Verfolgungsdrucks nicht mit Sicherheit aus.

Die Beigeladene beantragt,

die Klage abzuweisen.

Sie meint, es handele sich um eine verfolgungsbedingte Veräußerung, da unumstößlich feststehe, dass der Kaufpreis unangemessen niedrig gewesen sei. Der einzige Hinweis, dass Steindorff zu einem früheren Zeitpunkt beabsichtigt habe, der Universität die Sammlung zu schenken, ergebe sich aus dem Schreiben von W. vom 21. August 1936. Es handele sich nur um eine diffuse, nie konkretisierte Absicht, und der etwaige Zeitpunkt bleibe vage. Jedenfalls 1936/37 sei Steindorff fest entschlossen gewesen, die Sammlung zu verkaufen. Die freudigen Äußerungen über den Erhalt von Sammlungsstücken könnten nicht als Verzicht angesehen werden.

Das Gericht hat Beweis erhoben durch Vernehmung von Herrn H. als Zeugen mit dem aus dem Sitzungsprotokoll vom 26. Mai 2011 ersichtlichen Ergebnis.

Wegen der weiteren Einzelheiten des Sachverhaltes und des Vorbringens der Beteiligten wird auf die Gerichtsakte und die von der Beklagten eingereichten Verwaltungsvorgänge verwiesen, die Gegenstand der mündlichen Verhandlung gewesen sind.

\section{Entscheidungsgründe}

Die zulässige Anfechtungsklage ist unbegründet, weil der angegriffene Bescheid rechtmäßig ist und die Klägerin nicht in ihren Rechten verletzt ( $§ 113$ Abs. 1 VwGO). Die Beklagte hat die fragliche Sammlung zu Recht auf die Beigeladene zurückübertragen, denn diese ist Berechtigte iSv §§ 1 Abs. 6, 2 Abs. 1 Satz 3, 3 Abs. 1 Satz 1 VermG. 
Die Anmeldung vermögensrechtlicher Ansprüche durch die Klägerin im Wege einer Präzisierung ihrer "Globalanmeldung" (ANM-3) war entsprechend den Anforderungen der Rechtsprechung des Bundesverwaltungsgerichtes wirksam, denn aus den in Bezug genommenen Akten des Staatsarchivs Leipzig ergaben sich konkrete Hinweise auf den Verkauf der Sammlung durch Steindorff (BVerwG, Urteil vom 28. November 2007 - 8 C 12.06 -, Buchholz $428 \S 30$ VermG Nr. 41).

Die Voraussetzungen des § 1 Abs. 6 VermG liegen vor. Danach ist das Gesetz entsprechend auf vermögensrechtliche Ansprüche von Bürgern und Vereinigungen anzuwenden, die in der Zeit vom 30. Januar 1933 bis zum 8. Mai 1945 aus rassischen, politischen, religiösen oder weltanschaulichen Gründen verfolgt wurden und deshalb ihr Vermögen infolge von Zwangsverkäufen, Enteignungen oder auf andere Weise verloren haben. Gemäß Satz 2 dieser Norm wird ein verfolgungsbedingter Vermögensverlust nach Maßgabe des II. Abschnitts der Anordnung BK/O (49) 180 der Alliierten Kommandantur vom 26. Juli 1949 (nachfolgend: REAO) vermutet. Für Rechtsgeschäfte in der Zeit vom 15. September 1935 bis zum 8. Mai 1945 - wie im vorliegenden Fall - ist die Vermutung nur durch den Beweis zu widerlegen, dass der Veräußerer einen angemessenen Kaufpreis erhalten hat, über diesen frei verfügen konnte und zudem entweder das Rechtsgeschäft seinem wesentlichen Inhalt nach auch ohne die Herrschaft des Nationalsozialismus abgeschlossen worden wäre oder der Erwerber in besonderer Weise und mit wesentlichem Erfolg den Schutz der Vermögensinteressen des Berechtigten oder seines Rechtsvorgängers wahrgenommen hat (Art. 3 Abs. 2 und 3 REAO). Dabei genügt es für die Widerlegung der Vermutung durch die Führung des Beweises des Gegenteils nicht, dass ein anderer möglicher Hergang des Geschehens dargetan wird. Es ist vielmehr der volle Beweis des Gegenteils zu führen (BVerwG, Urteil vom 26. November 2003 8C 10.03 -, BVerwGE 119, 232 = juris Rdnr. 52). Dieser Beweis ist der Klägerin nicht gelungen.

Für den vorliegenden Fall gilt nicht deshalb etwas anderes, weil es sich bei der Veräußerung zu einem unter dem tatsächlichen Wert der Sammlung liegenden Kaufpreis um eine gemischte Schenkung gehandelt hätte, bei der ggf. Art. 4 REAO anzuwenden wäre (dafür Neuhaus in: Fieberg/Reichenbach, VermG § 1 Rdnr. 152; Godin Art. 5 Anm. 2; dagegen, aber letztlich mit gleichem Ergebnis Harmening u. a. Art. 4 Anm. 7). Dabei reicht die Feststellung, dass ein bewusster Preisnachlass vorlag, nicht aus, um eine gemischte Schenkung anzunehmen. Ansonsten würde die gesetzliche Systematik, wonach ein nachweislich unangemessener Kaufpreis zwingend - mangels Widerlegung der Vermutung - zur Annahme einer verfolgungsbedingten Veräußerung führt, ausgehebelt. Es muss daher zunächst festgestellt werden, dass tatsächlich eine Teilschenkung und nicht etwa ein - zwingend verfolgungsbedingter - Preisdruck vorlag. Art. 3 und 4 REAO enthalten dazu keine Regelung, sondern setzen die Feststellung, ob es sich um eine entgeltliche oder unentgeltliche Veräußerung handelte, voraus. Es ist daher von demjenigen, der sich darauf beruft, der volle Beweis dafür zu führen, dass es sich um einen schenkweisen Preisnachlass handelte. Das ist hier die Klägerin. Dabei mag es zwar sein, dass Steindorff "seiner" Universität die Sammlung zu einem schenkweise reduzierten Preis überlassen hat, bewiesen ist dies jedoch zur Überzeugung des Gerichtes nicht. So ist weder den Äußerungen von Steindorff selbst noch dem Schreiben von W. vom 28. Oktober 1936 an den Rektor mit hinreichender Sicherheit zu entnehmen, dass Steindorff bei einem Verkauf der Sammlung nach Hannover einen höheren Preis hätte erzielen können. So kann dem Schreiben von W. nicht entnommen werden, ob er hier zuverlässig einen - in Form eines Preisnachlasses - weiter bestehenden Schenkungswillen wiedergibt, oder ob er dem Rektor lediglich eine günstige Gelegenheit schmackhaft machen wollte. Erst Recht lag keine bereits 1908 vollzogene Schenkung vor, wie sich bereits daraus ergibt, dass ein Verkauf tatsächlich durchgeführt wurde, ohne dass sich die Universität darauf berufen hätte, ohnehin schon Eigentümer zu sein. Zudem sind die entsprechenden Äußerungen von $\mathrm{H}$., wie auch seine Zeugenvernehmung ergab, so zu verstehen, dass Steindorff der Universität lediglich den Besitz überlassen hatte, sich selbst aber weiter als verfügungsberechtigt ansah. Daher müsste die Klägerin die Vermutung des § 1 Abs. 6 Satz 2 VermG, Art. 3 Abs. 2 und 3 REAO widerlegen.

Es ist nach Auffassung des Gerichtes nicht mit hinreichender Sicherheit belegt, dass ein angemessener Preis vereinbart wurde. Zwar erscheint das sachverständig unterlegte Vorbringen der Klägerin, Abschläge im hier vorliegenden Umfang von ca. $20 \%$ seien beim Verkauf kompletter Sammlungen üblich, plausibel, doch genügt diese Plausibilität zur Überzeugungsbildung vor allem deshalb nicht, weil W. in dem genannten Schreiben erklärte, der tatsächliche Wert der Sammlung habe höher gelegen als die von Steindorff veranschlagten 10.260 RM, der Preis sei mäßig und Steindorff mit der Kaufpreisforderung bewusst unter dem tatsächlichen Wert geblieben. Auch dass es für die Motivation dieser Angaben wiederum die plausiblen Erklärungen gibt, die eine verfolgungsneutrale Preisbildung möglich erscheinen lassen, nämlich dass W. den Preis gegenüber vorgesetzten Behörden als besonders günstig darstellen wollte, begründet keine Überzeugungsgewissheit.

Der Kaufpreis dürfte frei verfügbar gewesen sein, denn maßgeblich ist der Zeitpunkt des Kausalgeschäfts auch dann, wenn der Kaufpreis nicht sofort zu zahlen war (BVerwG, Urteil vom 13. Dezember 2006 - 8 C 3.06 -, Buchholz $428 \S 1$ Abs. 6 VermG Nr. 39 = juris Rdnr. 29). Da jedenfalls die beiden ersten Kaufpreisraten Steindorff offenbar ungestört zugeflossen sind, besteht kein Anlass anzunehmen, dass er in seiner freien Willensbildung in der Form beeinflusst war, dass er veräußert hat, obwohl erkennbar war, dass er den Kaufpreis nicht zur freien Verfügbarkeit werde erhalten können. Im Übrigen dürfte das Schreiben der Zollfahndungsstelle vom 2. April 1940 zeigen, dass der Schwiegersohn die letzte Rate im Wege der Aufrechnung mit Steindorff vorgestreckten Ausgaben erhalten hat. 
Jedenfalls aber hat die Klägerin nicht die bei Rechtsgeschäften nach dem 15. September 1935 greifende verschärfte Vermutung des Art. 3 Abs. 3 REAO widerlegt. Der Klägerin ist zwar zuzugestehen, dass sie ein in sich stimmiges und auch plausibel erscheinendes Szenario dargestellt hat, dass ein verfolgungsneutrales Geschäft als möglich erscheinen lässt. Dies wäre aber nur geeignet, etwa einen für die Verfolgung sprechenden Anscheinsbeweis zu erschüttern. Das hier erforderliche Beweismaß wird dagegen verfehlt, denn wie oben ausgeführt ist zur Widerlegung der hier zu Gunsten der Beigeladenen streitenden Verfolgungsvermutung der volle Beweis des Gegenteils zu führen.

Dabei mag sich den vorliegenden Unterlagen über die Verkaufsverhandlungen zunächst entnehmen lassen, dass Steindorff zu einem früheren Zeitpunkt, der mit hoher Wahrscheinlichkeit nicht nur vor der Machtergreifung, sondern sogar vor der Weltwirtschaftskrise gelegen haben dürfte, die Absicht hatte, seine Sammlung der Universität zu schenken. Dem ist aber schon nicht zu entnehmen, für welchen Zeitpunkt er eine Schenkung ins Auge gefasst hatte; denkbar wäre insbesondere ein Vermächtnis, so wie er auch den Zettelkatalog zu seiner Gisa-Ausgrabung erst nach seinem Tod einem Kollegen, ersatzweise der Universität zukommen lassen wollte. Die weiteren, von W. wiedergegebenen Äußerungen, er habe die Sammlung als "Sparbüchse“ angesehen, lassen sogar die Vermutung nahe liegend erscheinen, dass er eine Veräußerung erst - wenn überhaupt - in fernerer Zukunft plante, also etwa wenn entweder Bargeldbedarf bestünde oder im Gegenteil die Lage sich entspannen würde.

Ein derartiger Bargeldbedarf lag vor, denn nach den vorliegenden Unterlagen wollte Steindorff die Sammlung auf jeden Fall, wenn auch bevorzugt an die Universität Leipzig, verkaufen. Dass eine anderweitige Veräußerung nur vorgeschoben gewesen wäre, ist nicht zu belegen. Ebenfalls nicht belegt ist, dass der nach dem Vorbringen der Klägerin aus der Geschäftsübernahme des Schwiegersohnes resultierende Geldbedarf verfolgungsneutral entstanden wäre. Dabei kann dahinstehen, ob die Verfolgungsbedingtheit - etwa in Form einer adäquaten Kausalität - bereits deshalb anzunehmen ist, weil die Gelegenheit zum Erwerb eines bislang jüdischen Rauchwarengeschäftes wiederum aus der Verfolgungssituation entstanden ist. Jedenfalls ist dies als alleiniger Grund für den Geldbedarf ebenfalls nicht bewiesen. Der enge zeitliche Zusammenhang zwischen Veräußerung der Sammlung und Erwerb des Unternehmens K. GmbH durch den Schwiegersohn belegt dies nicht, denn die Schlussfolgerungen der Klägerin sind nicht zwingend. Zunächst besteht eine deutliche Diskrepanz zwischen dem Preis der Sammlung und der Höhe der - nach Umwandlung in eine KG - ausgewiesenen Kommanditeinlage von 130.000 RM. Zwar war der Schwiegersohn nicht der Kommanditist, sondern der Komplementär, doch reicht dieser Hinweis auf das Gesamtvolumen des Geschäfts aus, die Frage aufzuwerfen, ob und inwieweit ein Betrag von 8.000 RM dabei hilfreich gewesen sein kann. Auch ist der Zusammenhang nicht durch den Zeitpunkt der Übernahme der Gesellschaft am 18. September 1936 belegt.
Zwar hatte Steindorff in seinem Angebot an die Universität eine Annahmefrist bis zum 15. September 1936 gesetzt, doch gibt es keinen Hinweis darauf, dass innerhalb dieser Frist tatsächlich eine wie auch immer geartete Zusage erfolgte. Jedenfalls eine verbindliche Zusage, auf die sich eine Investitionsentscheidung des Schwiegersohnes hätte stützen können, kann nicht erfolgt sein, da die endgültige Genehmigung erst am 1. März 1937 erfolgte und vorher kein Geld geflossen ist.

Etwas Anderes ergibt sich auch nicht aus der Zeugenaussage. Zwar hat der Zeuge bestätigt, dass Steindorff seinem Schwiegersohn Geld für den Erwerb eines Rauchwarenhandels zur Verfügung gestellt hat. Er hat aber ebenfalls bekundet, dass sich Steindorff seit seiner Emeritierung finanziell einschränken musste, so dass nicht belegt ist, dass der Finanzbedarf allein durch die Unternehmung des Schwiegersohnes begründet war. Weiterhin hat der Zeuge bekundet, dass sich Steindorff einerseits zunächst nicht bedroht gefühlt und den Rat, von einer Vortragsreise nicht nach Deutschland zurückzukehren, nicht befolgt habe. Andererseits sei er aber nur deshalb nach Deutschland zurückgekehrt, um ein wichtiges wissenschaftliches Werk mit den in Leipzig zur Verfügung stehenden Mitteln vollenden zu können. Diese ungenauen Angaben lassen nicht den Schluss zu, dass Steindorff sich auch noch zum Zeitpunkt des Verkaufes der Sammlung sicher gefühlt und etwa erst durch die Novemberpogrome zur Auswanderung bewegt worden wäre. Ebenso wahrscheinlich ist der Schluss, dass Steindorff bereits zu diesem Zeitpunkt Vorbereitungen für seine nach Abschluss des genannten Werkes vorgesehene Auswanderung traf. Schließlich benötigte er nachweislich die letzte Rate für Auswanderungskosten.

Angesichts dessen kann keine Rede davon sein, dass eine Verfolgungsbedingtheit widerlegt wäre, weil es vielmehr nicht unwahrscheinlich erscheint, dass zumindest der Zeitpunkt der Veräußerung auch durch die politischen Umstände beeinflusst war.

Auch ausgehend von der Annahme, es habe sich um eine gemischte Schenkung gehandelt, wäre die Klage unbegründet. Wie der Fall der gemischten Schenkung letztlich zu beurteilen ist, ist der rückerstattungsrechtlichen Rechtsprechung nicht zu entnehmen. Nach Neuhaus sind offenbar der Kaufteil und der Schenkungsteil getrennt zu betrachten mit der Folge, dass die Verfolgungsbedingtheit nur eines von beiden Teilen in Betracht kommt (aaO Rdnr. 153). Dagegen spricht, dass es sich bei einer gemischten Schenkung nicht um zwei nebeneinander stehende Geschäfte handelt, sondern um einen einheitlichen Vorgang, nämlich um eine Veräußerung zum Vorzugspreis, die wegen ihres Mischcharakters beiden Regelungskomplexen unterfällt (vgl. auch RG, Urteil vom 27. Juni 1935 - IV 28/35 -, RGZ 148, 236). Selbst wenn also - unter Anwendung von Art. 4 REAO - festgestellt werden sollte, dass der Preisnachlass verfolgungsneutral war, steht damit ggf. fest, dass der Kaufpreis nicht unangemessen niedrig iSv Art. 3 Abs. 2 REAO war; über sonstige für oder gegen die Verfolgungsbedingtheit sprechende Ge- 
sichtspunkte ist damit aber noch nichts gesagt (Harmening u.a. Art. 4 Anm. 7; so wohl auch Wasmuth in: RVI, VermG § 1, Rdnr. 220). Es ist daher jedenfalls hinsichtlich des Kaufteiles des Geschäfts Art. 3 REAO anzuwenden; da ein verfolgungsbedingter Verkauf notwendig auch die logisch nachfolgende Schenkung in Form des Forderungserlasses infiziert, kommt es nicht darauf an, ob die Vermutung des Art. 3 REAO nur für den Kaufanteil des Vertrages oder auch für den Schenkungsanteil gilt. Wenn dem Art. 4 REAO eine Entziehungsvermutung innewohnt, für die die Beschränkung der Widerlegungsmöglichkeit nach Art. 3 Abs. 2 und 3 REAO nicht gilt, wäre für diesen Teil zwar der direkte Gegenbeweis eröffnet (so OLG Hamburg vom 10. März 1951 - 5 W 21/51 -, RzW 1951, 171, und vom 31. Juli 1954 - 5 WiS 151-153, 177, 209/54 -, RzW 1955, 166; OLG Köln vom 2. Oktober 1953 - 7 RW 97/53 -, RzW 1953, 353; Küster RzW 1949/50, 176), nicht aber für das Geschäft insgesamt. Insoweit kann die Verfolgungsvermutung grundsätzlich nur durch die in Art. 3 Abs. 2 und 3 REAO vorgesehenen Beweise widerlegt werden (BVerwG, Urteil vom 30. April 2003 - 8 C 9.02 -, Buchholz 428 § 1 Abs. 6 VermG Nr. 18 = juris Rdnr. 23 mwN). Auch wenn es dann nicht auf die Angemessenheit des Kaufpreises ankommen sollte, müsste die Klägerin immer noch beweisen, dass das Rechtsgeschäft auch ohne die Herrschaft des Nationalsozialismus abgeschlossen worden wäre. Diesen Beweis kann sie aber - wie oben ausgeführt - nicht führen.

Ein Verzicht auf Wiedergutmachung, den sich die Beigeladene entgegenhalten lassen müsste, liegt nicht vor. Ein solcher ist dann in Betracht zu ziehen, wenn im Zusammenhang mit Wiedergutmachungsleistungen Erklärungen vom Geschädigten abgegeben worden sind, die als unwiderruflicher Verzicht auf alle bestehenden und künftigen Ansprüche wegen des erlittenen Vermögensverlusts auszulegen sind. In einer solchen Erklärung müsste zudem ein rechtlich bedeutsames Verhalten im Blick auf zukünftige Ansprüche liegen, insbesondere auf solche, die sich nach einer Wiedervereinigung Deutschlands ergeben könnten (BVerwG, Urteil vom 27. Mai 1997 - 7 C 67.96 -, Buchholz $428 \S 1$ VermG Nr. 112 = juris Rdnr. 16 f. mwN). Zwar ist den Schreiben von Steindorff aus der Nachkriegszeit sehr wohl Freude über die Wiederaufstellung der Sammlung zu entnehmen. Es ist ihnen jedoch nicht zu entnehmen, dass diese Freude sich auf mehr bezog als auf den Stolz über die erneuerte Anerkennung seines Lebenswerkes und darüber, dass seine Sammlung nicht völlig im Krieg zerstört wurde oder der Öffentlichkeit verschlossen blieb. Angesichts der damals mangels entsprechender Regelungen in der SBZ und DDR fehlenden Möglichkeit, Rückgabeansprüche geltend zu machen, lässt sich daraus nicht mit hinreichender Gewissheit ableiten, dass er auf eine Rückgabe auch dann verzichtet hätte, wenn es eine realistische Aussicht darauf gegeben hätte. Gleiches gilt für die Anmeldung von Ansprüchen nach dem Bundesrückerstattungsgesetz nur für andere Vermögenswerte, denn es ist nicht auszuschließen, dass die Anmeldung hinsichtlich der Sammlung bereits deshalb unterblieb, weil sie - anders als die Silberwaren, bei denen dies ungewiss erscheinen konnte - nachweislich nicht in den Geltungsbereich dieses Gesetzes gelangt waren.
Soweit H. als (mutmaßlicher) Rechtsnachfolger von Steindorff bekundet hat, keine Ansprüche geltend machen zu wollen, um der Klägerin die streitige Sammlung belassen zu können, ist dies ebenfalls unerheblich, und zwar bereits deshalb, weil er keine Ansprüche innerhalb der Frist des $\S 30$ a Abs. 1 VermG angemeldet hat. Zudem ist der Wortlaut des § 2 Abs. 1 Satz 3 VermG, der nicht auf die formelle Anmeldung von Restitutionsansprüchen abstellt, sondern die Klägerin als Rechtsnachfolgerin einsetzt, "soweit Ansprüche ... nicht geltend gemacht werden", dahin zu verstehen, dass die Rechtsnachfolge auch solche Ansprüche von Berechtigten umfasst, die zwar zunächst angemeldet, im Fall einer negativen Bescheidung aber nicht durchgesetzt wurden. Nach dem Gesetzeswortlaut ist die Rechtsnachfolge der Klägerin fingiert, sie "gilt" nur als Rechtsnachfolger. Sie muss sich deshalb auch nicht den Verzicht eventueller Berechtigter auf die weitere Durchsetzung ihrer Ansprüche durch Antragsrücknahme oder Verzicht auf Rechtsmittel gegen ablehnende Bescheide zurechnen lassen. Nur so kann dem Zweck der Rechtsnachfolgeregelung zugunsten der Klägerin, dass - wenn die Restitutionsansprüche nicht jüdischen Berechtigten oder ihren Rechtsnachfolgern zu Gute kommen - nicht der Fiskus des Staates begünstigt werden soll, in dessen Geschichte sich das wieder gutzumachende Unrecht ereignet hat (vgl. BT-Drs. 11/7831 S. 4), umfassend Rechnung getragen werden (BVerwG, Urteil vom 28. April 2004-8 C 12.03 -, BVerwGE 120, $362=$ juris Rdnr. 26).

Das Gericht verkennt nicht, dass es unbefriedigend erscheinen mag, wenn in Folge dieser Entscheidung die Sammlung der Öffentlichkeit und insbesondere dem wissenschaftlichen Nachwuchs entzogen werden sollte. Für derartige Erwägungen wäre nur im Rahmen der Restitutionsausschlussgründe nach $\S$ 4, 5 VermG Raum, die jedoch für bewegliche Kulturgüter keine Regelung treffen.

Die Kostenentscheidung beruht auf $\S \S 154$ Abs. 1, 162 Abs. 3 VwGO. Es entspricht der Billigkeit, der Beigeladenen einen Kostenerstattungsanspruch zuzuerkennen, da sie einen Antrag gestellt hat und damit ein eigenes Kostenrisiko eingegangen ist (vgl. § 154 Abs. 3 VwGO).

Die Berufung gegen dieses Urteil ist gemäß $\S 37$ Abs. 2 VermG ausgeschlossen. Gründe, gemäß §§ 132, 135 VwGO die Revision zuzulassen, liegen nicht vor. Rechtsfragen der Verfolgungsvermutung und deren Widerlegung nach $\S 1$ Abs. 6 VermG iVm Art. 3 REAO sind in der höchstrichterlichen Rechtsprechung umfassend geklärt. Der vorliegende Fall bietet keine neuen rechtlichen Aspekte. Insbesondere kommt es nicht auf höchstrichterlich nicht geklärte Rechtsfragen im Zusammenhang mit gemischten Schenkungen an. (Entscheidung von der Redaktion bearbeitet.) 